\title{
36V/400Hz Three-phase Soft-switching Power Supply Design
}

\author{
Yalong Liu, a ,Guanyue Zhang ${ }^{1, b}$ and Kuangcheng $\mathrm{Li}^{1, \mathrm{c}}$ \\ ${ }^{1}$ Academy of Armored Force Beijing China \\ anottinghillove@163.com, ${ }^{b}$ arthlonliu@189.cn, likuangcheng@163.com
}

\begin{abstract}
Keywords: Inverter DPM Soft-switching High-frequency pulsed DC link
Abstract: This article describes the design process of a three-phase AC soft-switching static power supply using discrete pulse modulation (DPM) technology. Firstly, the principle diagram of the static power supply of the combined inverter structure is given. The working principle of the main circuit of the soft-switching inverter and the control strategy of the static power supply are analyzed. Based on this, the experimental waveforms are given. The characteristics of the power supply are: no rotating parts, low noise; the use of high-frequency power conversion technology to reduce the size and weight of the static power supply itself; the application of soft-switching technology in the main circuit of the power conversion makes the electromagnetic compatibility of the static power supply Get further improvement.
\end{abstract}

\section{Introduction}

The $400 \mathrm{HZ}$ static power supply is a converter device that converts DC power into $400 \mathrm{HZ}$ AC power. The output voltage is mainly in two types: $115 \mathrm{~V}$ and $36 \mathrm{~V}$. Most of the special vehicles are powered by DC generators or DC batteries, which are used as secondary power supply after inverters. At present, special vehicles use rotating current transformers to provide $36 \mathrm{~V} / 400 \mathrm{HZ}$ three-phase AC power. The rotary converter consists of a DC motor, a three-phase alternator and a frequency stabilizer. The disadvantage of this power supply mode is that the power conversion efficiency is low and the operation noise is large. With the development of power electronics and power switching devices, the static power supply has become an ideal alternative power supply for rotating converter devices [1]. At present, more advanced inverters are being developed in the direction of surface mounting and other aspects of the process; control focuses on multi-loop control, digital control and other methods, and the use of high-frequency soft-switching technology. This article describes the design of a $36 \mathrm{~V} / 400 \mathrm{HZ}$ three-phase AC soft-switching static power supply using discrete pulse modulation (DPM) technology.

\section{Static power supply structure and main circuit design}

Figure 1 shows the block diagram of the static power supply using this structure. The static power supply is based on a single-phase high-frequency pulsed DC link inverter module and uses a combined inverter structure. Each phase inverter module is independent of each other, the difference being that the reference sine wave in the control circuit of each module has a phase difference of $120^{\circ}$. The grounds of the three single-phase inverter modules are connected together as a neutral output. The advantage of this kind of three-phase AC static power supply is that it has a strong ability to carry unbalanced loads; each phase can be independently controlled and the control circuit is simple; a modular structure is achieved and the system reliability is high. 


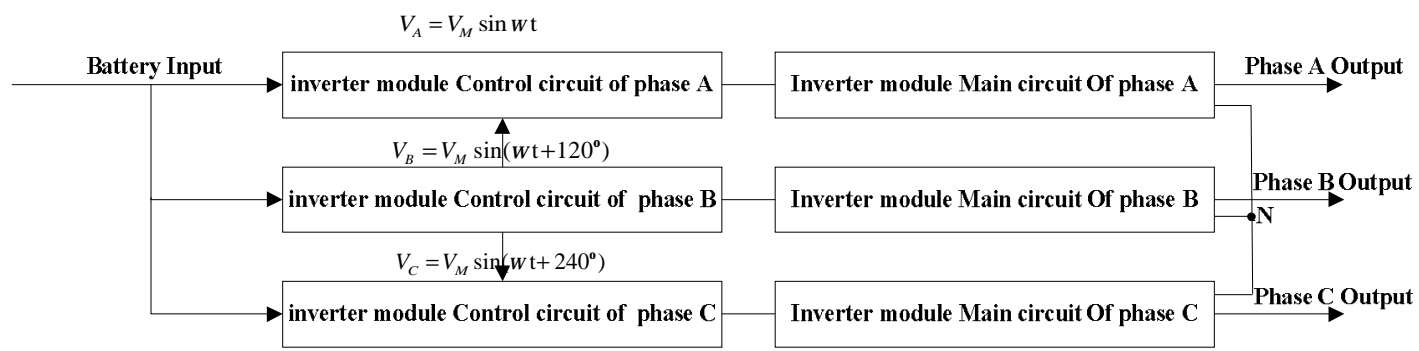

Fig.1 Block diagram of three-phase high-frequency pulsed DC link static power supply with modular inverter structure

Figure 2 shows a block diagram of a single-phase inverter, using DPM-controlled high-frequency pulsed DC link soft-switching technology. The DC/DC conversion link realizes soft switching through the resonance technology, and the inverting link realizes soft switching through the high-frequency pulsed DC conversion technology to reduce the switching loss. The power conversion stage is: battery DC input -DC/DC chopper - high frequency pulse DC - DC/AC inverter $-36 \mathrm{~V} / 400 \mathrm{HZ}$ AC.

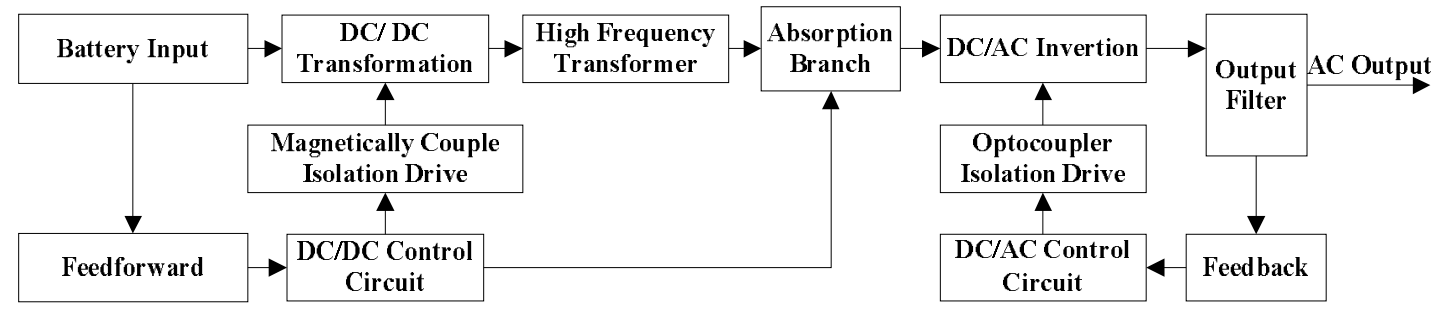

Fig. 2 Single-phase inverter module block diagram

The main circuit of the soft-switching inverter designed based on the block diagram is shown in Figure 3. The dual-circuit forward circuit is used as the main circuit topology of the DC/DC link. The purpose is to realize the voltage matching of the intermediate circuit and generate the pulsed DC voltage. The main control pipe is the switch $S_{1}$ and $S_{2}$. The high-frequency transformer $T_{1}$ achieves the isolation of input and output. The DC/AC link uses a full-bridge inverter circuit as the main circuit topology. The output $\mathrm{u}_{\mathrm{CD}}$ of the DC/DC converter is a pulsed direct current with a certain duty cycle. The on-off of the switching transistors $\mathrm{Q}_{1} \sim \mathrm{Q}_{4}$ on the bridge arm is controlled during the zero phase of the pulsed voltage $\mathrm{u}_{\mathrm{CD}}$ to ensure that the source/drain voltage is zero. The on-off switching of the switch tube is achieved so that a zero-voltage switch can be reliably realized. The switching timing of the switching legs of the inverter bridge arm is controlled, and the output voltage $\mathrm{u}_{\mathrm{AB}}$ is generated at the midpoint of the two bridge arms. After the LC output filtering section, the $400 \mathrm{HZ}$ single-phase alternating current electric circuit $\mathrm{u}_{\mathrm{o}}$ is formed.

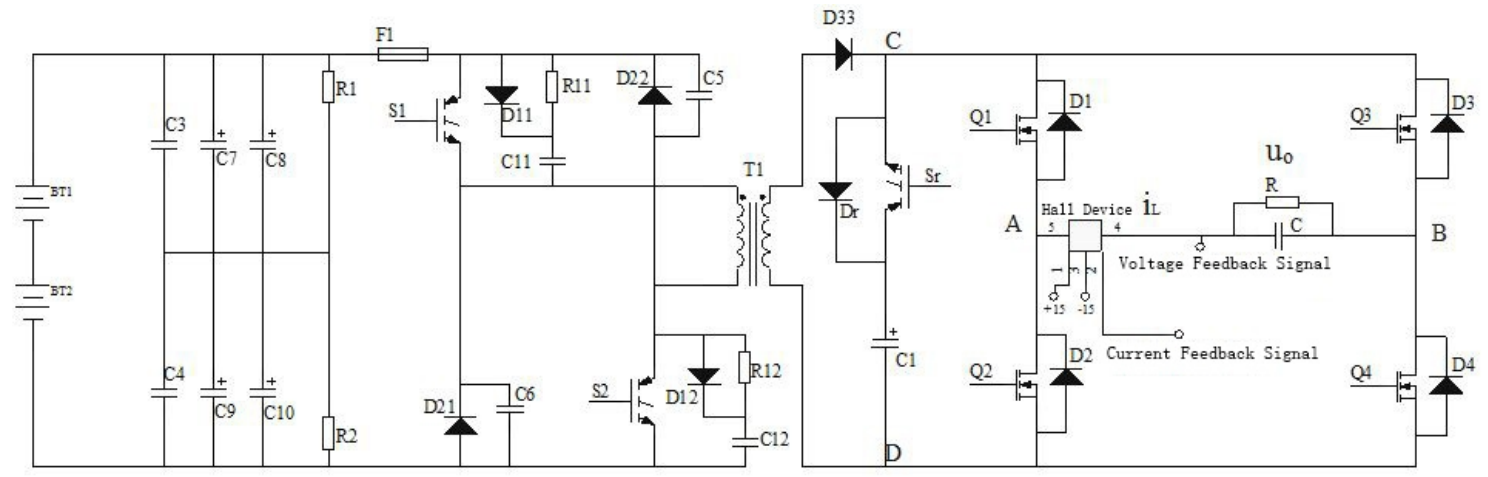

Figure 3 Soft switching inverter main circuit

In order to meet the requirement of soft switching, the switching point of the control pulse of the switch tube on the inverter arm is discontinuous on the time axis, so discrete pulse modulation (DPM) is adopted. Figure 4 shows the principle of the inverter bridge under discrete pulse modulation. The working state of the inverter bridge is as follows (from A to B are positive 
directions): the current feedback signal if is compared with the current given signal $\mathrm{i}_{\mathrm{g}}$ ( $400 \mathrm{HZ}$ sine wave signal), when if exceeds the hysteresis lower limit $\mathrm{i}_{\mathrm{g}}-\mathrm{a}, \mathrm{Q}_{1}, \mathrm{Q}_{4}$ is on, the output modulation voltage $u_{A B}$ is equal to $u_{C D}$, and the output current $i_{L}$ rises. This state is called +1 state, that is, the DC voltage supply state; when $i_{f}$ is within the hysteresis range $\left(i_{g}-a, i_{g}+a\right), Q_{2}, Q_{4}$ is on, $Q_{1}$ and $Q_{3}$ are off, the output voltage $u_{A B}$ of the bridge arm is equal to 0 , and the output inductor current $i_{L}$ is freewheeling through $\mathrm{D}_{2}, \mathrm{Q}_{4}$ or $\mathrm{D}_{4}, \mathrm{Q}_{2}$, and $\mathrm{i}_{\mathrm{L}}$ falls. This state is called 0 state, ie freewheeling state. When $i_{f}$ exceeds the hysteresis limit $i_{g}+a, Q_{2}$ and $Q_{3}$ turn on and the output voltage $u_{A B}$ equals $-u_{C D}$. The output current $i_{L}$ increases in reverse. This state is called the -1 state. Therefore, the midpoint voltage of the two arms has three states: $\mathrm{u}_{\mathrm{CD}},-\mathrm{u}_{\mathrm{CD}}$, and 0 . This satisfies the phenomenon that the inverter arm is in the unipolar operation mode, and the no-function feedback is small, so as to avoid the phenomenon that the pulse voltage does not return to zero.

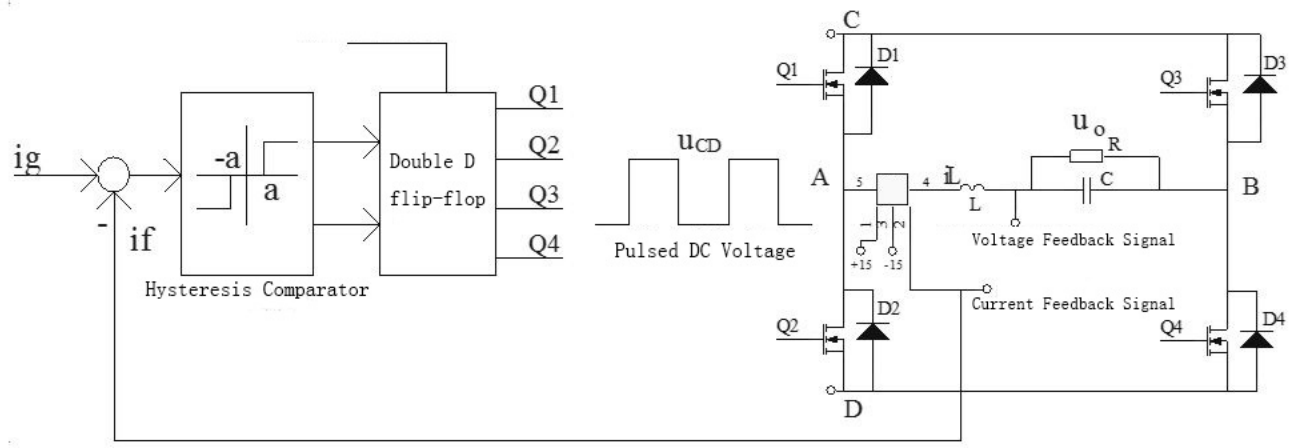

Fig. 4 Schematic diagram of three-state discrete pulse modulation of inverter bridge

\section{Static Power Control Strategy Design}

\section{Expansion of Duty Cycle of DC/DC Converter}

The DC/DC conversion section of the single-phase inverter module adopts input voltage feed-forward control. The change of the battery input voltage and load affects the duty cycle of the PWM control chip output, so as to ensure the stability of the average value of the output pulse voltage of the DC/DC conversion section. In order to overcome the defect that the duty cycle of the forward circuit cannot be too large, an absorption circuit composed of a switching transistor $\mathrm{S}_{\mathrm{r}}$ and a high-frequency electrolytic capacitor $\mathrm{C}_{1}$ is added in the inverter to achieve an extension of the duty ratio. The control pulse is relative to the switching transistors $S_{1}$ and $S_{2}$. The control pulse delays turn-on and delays turn-off. During $S_{1}$ and $S_{2}$ off, due to the delay of $S_{r}$ turning off, $C_{1}$ discharges to the full-bridge inverter, increasing the duty cycle of the pulsed DC voltage and improving the power conversion efficiency.

\section{DC/AC transformation link control strategy design}

The DC/AC converter uses three-state hysteresis current control. The use of instantaneous voltage and current feedback form a double closed-loop adjustment. The inverter control circuit consists of a reference sine wave generation circuit, a voltage loop regulator circuit, a current hysteresis comparison circuit, and a sample-and-hold circuit. The outer ring is a voltage loop. The PI regulator is used to take the output voltage as the feedback signal. The $400 \mathrm{HZ}$ sine wave ur generated by the reference sine wave circuit is used as the voltage regulator given signal. The inner ring is a current loop, taking the output current as a feedback signal, and the output of the PI regulator of the voltage loop as a given signal, and a three-state hysteresis regulator is used to regulate the output current [4]. Figure 5 shows the block diagram of the transfer function of the inverter. 


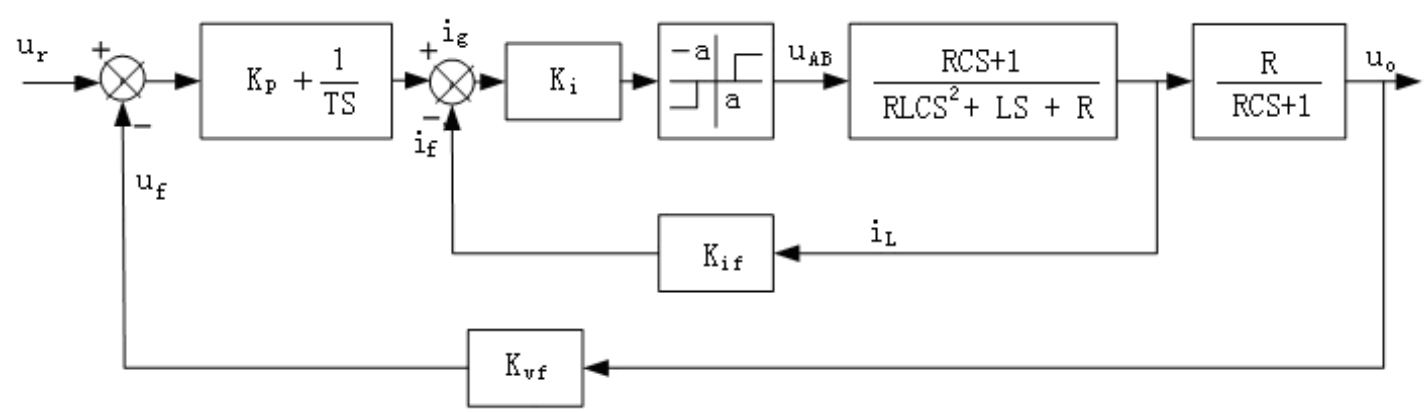

Fig. 5 Inverter transfer function block diagram

The current inner loop has a natural current limiting function, which allows the inverter to limit the maximum output current when the output is short-circuited, and is also conducive to improving the dynamic response of the system. The voltage loop ensures that the output voltage has good sinusoidal, less harmonic content, and harder external characteristics. In Figure 5, ur is the reference sine wave reference signal; $\mathrm{u}_{\mathrm{f}}$ is the feedback signal after the output voltage from both ends of the filter capacitor $\mathrm{C}$ is divided; $\mathrm{K}_{\mathrm{p}}$ is the proportional coefficient of the voltage regulator; $\mathrm{K}_{\mathrm{vf}}$ is the voltage feedback coefficient; $i_{g}$ is the current Fixed signal; $i_{L}$ is the inductor current; if is the current feedback signal generated after the $i_{L}$ flows through the Hall device; $K_{i f}$ is the current feedback coefficient. The three-state hysteresis regulator makes the feedback current if track the $i_{g}$ variation within the width a of the positive and negative hysteresis loops. After the hysteresis loop comparison result is sampled and maintained, the output voltage $\mathrm{u}_{\mathrm{AB}}$ of the inverter arm midpoint is controlled. After the $u_{A B}$ is filtered by the output filter, the final required voltage $u_{o}$ is obtained.

\section{Inverter bridge switch tube soft switch implementation}

Figure 6 shows the schematic diagram of the inverter bridge arm soft-switching. Under the control of the driving pulse at a certain switching timing, the two-transistor forward circuit generates a pulsed DC voltage with a constant pulse width between two points of the absorption branch $\mathrm{C}$ and $\mathrm{D}$. The pulse signal is input to the control circuit of the inverter circuit by sampling the isolation transformer, and the signal is subjected to reverse shaping to form the output of the clock pulse CLK to the control switch pulse of the inverter link (output of the double D flip-flop in FIG. 4). Control. The output of the D flip-flop can only change when the clock signal is high. The soft-switching operation state is analyzed according to the timing shown in FIG. 6: At the time $\mathrm{t}_{7}$, the output of the hysteresis comparator has jumped, but since the clock signal is still low, the output of the D-flip-flop and the output of the switch driving circuit are both No change occurs. At time $\mathrm{t} 1$, the clock pulse is a high-level signal, and the change of the output of the hysteresis comparator is reflected on the output of the D-type flip-flop. As shown in the figure, the drive signal $\mathrm{u}_{\mathrm{GS}}$ only changes at $\mathrm{t}_{5}$. The shutdown process is similar to this. Therefore, the switching tube driving pulse drives the switching tube when the voltage between $\mathrm{C}$ and $\mathrm{D}$ is zero, that is, the switching state of the switching tube on the inverter arm can only be changed during the period when the voltage of the pulsed DC link is zero. Realize the soft switch of the switch tube $\mathrm{Q}_{1^{-}} \mathrm{Q}_{4}$. 


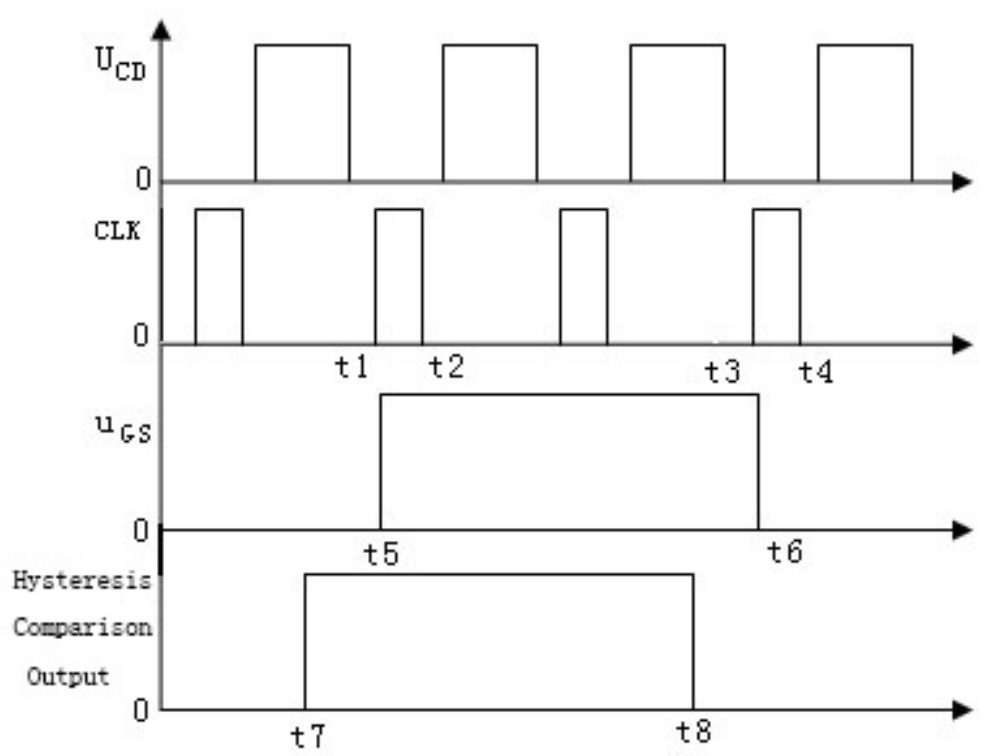

Fig. 6 Inverter bridge soft switch schematic

\section{Generation of a reference sine wave for a stationary power supply}

The three-phase reference sine wave generator consists of three single-phase reference sine wave generators that achieve a phase difference of $120^{\circ}$ between the output voltages of the single-phase modules.

Single-phase reference sine wave generator is composed of frequency division circuit, a staircase wave synthesis circuit, and an active filter circuit. The oscillating circuit includes a $3.6864 \mathrm{M}$ crystal oscillator and a chip CD4060. Its 9-pin output clock signal is 7.2K. The staircase wave synthesizing circuit includes two presettable counters CD4018, nine weighting resistors, and one inverter. The circuit synthesizes the clock signal and outputs a sine wave with an order of 18 after the combination of the cyclic shift and the weighted resistance. After active filtering, the $400 \mathrm{~Hz}$ reference sine wave $\mathrm{u}_{\mathrm{r}}$ is output.

The three staircase wave synthesizer circuits in the three-phase reference sine wave generator commonly use a clock signal generated by an oscillating frequency dividing circuit, and the phase difference of the reference sine wave in each single-phase module control circuit is $120^{\circ}$ using the preset number function of the chip CD4018. The figure is shown in Figure 7. When the three-phase static power supply control circuit works, the differential circuit composed of $\mathrm{C} 1$ and R1 provides the initial set high level for the set allowable end 'PE' and the CD4018 counter set number, and its value is set by 'J1' 'J9'. High and low level decision. The 18th-order staircase wave corresponds to $360^{\circ}$, and the three-phase reference sine wave has a phase difference of $120^{\circ}$. That is, each 6th step wave corresponds to a $120^{\circ}$ phase relationship, and the preset of the chip CD4018 in each phase sine wave generator shown in FIG. 7 is set. value. The preset number of each phase is not unique, as long as the phase relationship is satisfied. 


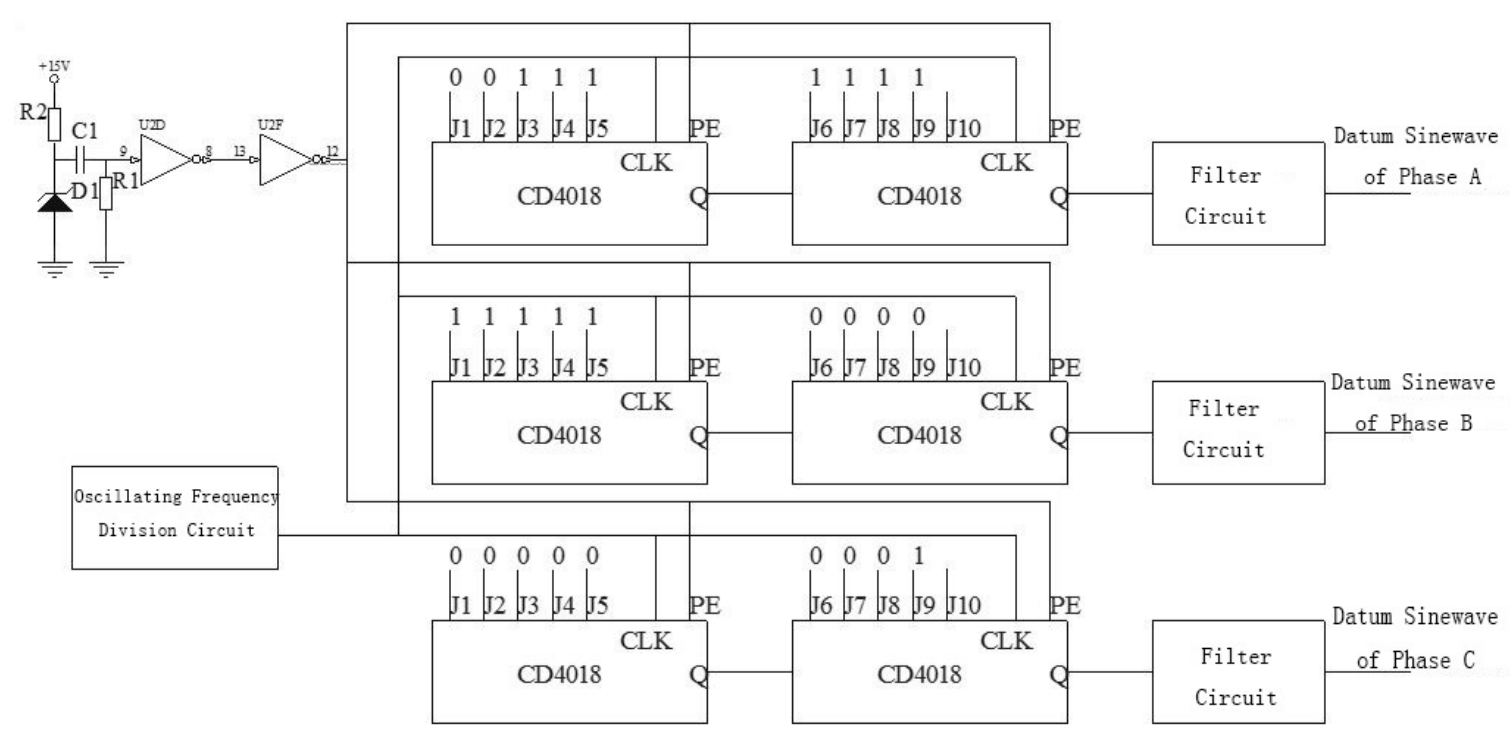

Fig. 7 Schematic diagram of three-phase reference sine wave generation

\section{Principle prototype and experimental waveform}

In the experiment, two 7-HK-182 lead batteries were used to supply $(28 \pm 4 \mathrm{~V})$, the duty ratio of the DC link was adjusted to 0.65 , and a pulsed DC voltage with a pulse frequency of $33 \mathrm{~K}$ was output on the secondary side of the high-frequency transformer. The output filter is $\mathrm{C}=15 \mathrm{uF}$ and $\mathrm{L}=0.7 \mathrm{mH}$. The inverter output is $36 \mathrm{~V} / 400 \mathrm{~Hz}$ alternating current, and the hysteresis width $\mathrm{a}=0.3 \mathrm{in}$ the control circuit.

Figure 7 shows the output of the three-phase AC static power supply and the soft-switching experimental waveform of the high-frequency transform soft-switching three-phase AC power supply. The output voltage waveform is good, and the switch tubes on the inverter bridge achieve zero voltage turn-on and turn-off (the test tube is A-phase module switch tube $\mathrm{Q}_{2}$, model IXFK48N50, and $\mathrm{u}_{\mathrm{Q} 2}$ are the gate drive voltage of the tube).

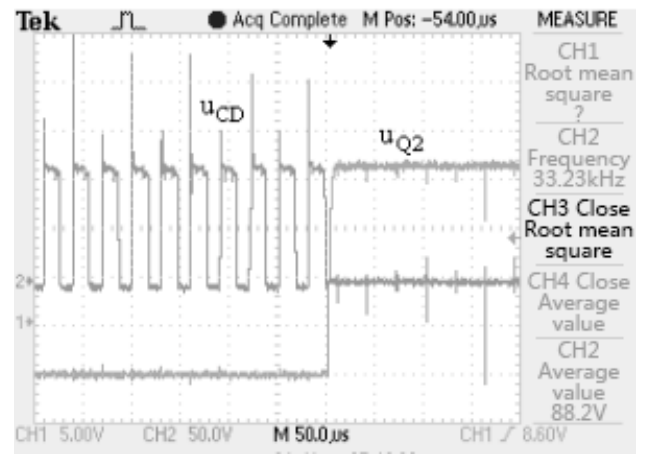

a Switch zero voltage turn-on waveform

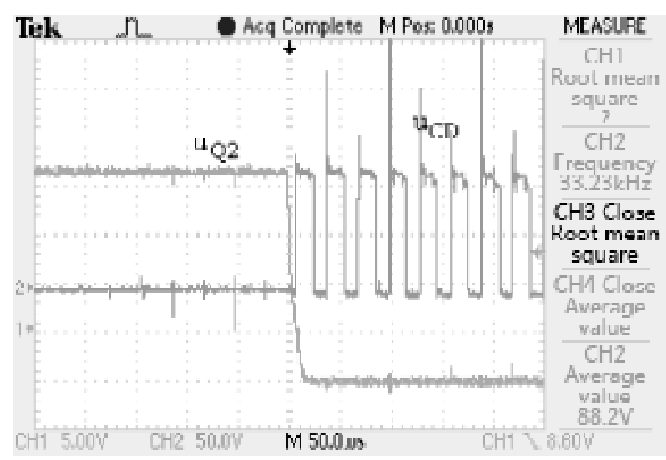

b Switch zero voltage off waveform

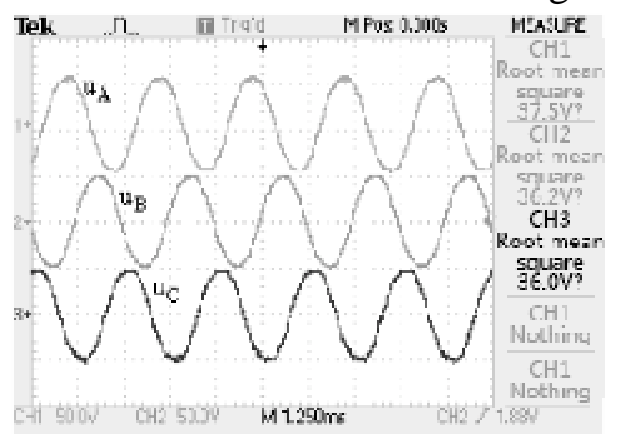

c Three-phase AC output waveform

Fig. 8 Soft switching inverter experiment waveform 


\section{Conclusion}

The experimental results show that under the three-state DPM current hysteresis control, the $36 \mathrm{~V} / 400 \mathrm{~Hz}$ three-phase AC soft-switching power supply based on the high-frequency pulsed DC link has good performance such as good stability and fast dynamic response. The switch tube on the inverter bridge arm realizes a soft switch and does not increase the auxiliary device to solve the problem of switching loss. The high quality of power supply can replace the existing rotary converter device to provide $36 \mathrm{~V} / 400 \mathrm{HZ}$ three-phase AC power supply.

\section{References}

[1]Zang Kemao,,Ma Xiaojun,,Li Changbing. Modern Tank Gun Control System. BeiJing: National Defence Industry Press

[2] Lin Weixun. Modern Power Electronics Technology. Beijing: Machinery Industry Press 2006

[3] Chen Daolian. DC-AC Inverter Technology and Application. Beijing: Machinery Industry Press 2005

[4] Maria D.Bellar ,Aristide Tchamdjiou, Javad Mahdavi and M.Ehsani. A Review of Soft-switched DC/AC Converters. IEEE Trans. on IA.1998 34 ( 4 ),847-860

[5] Naser M.Abdel-Rahim, John E.Quaicoe.Analysis and Design of a Multiple Feedback Loop Control Strategy for Single-Phase Voltage-Source UPS Inverters. IEEE Trans on PE. 199611 ( 4 ) : 532-538. 